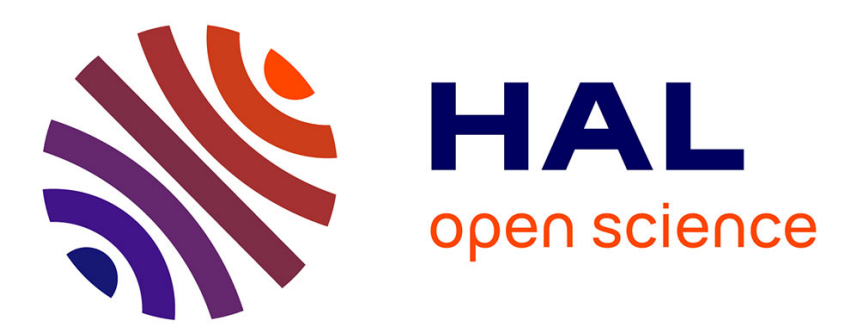

\title{
Multirate technique for explicit Discontinuous Galerkin computations of time domain Maxwell equations on complex geometries
}

\author{
Abelin Kameni, Bruno Seny, Lionel Pichon
}

\section{- To cite this version:}

Abelin Kameni, Bruno Seny, Lionel Pichon. Multirate technique for explicit Discontinuous Galerkin computations of time domain Maxwell equations on complex geometries. IEEE Transactions on Magnetics, 2016, 52 (3), pp.7204604. 10.1109/TMAG.2015.2480537 . hal-01235050

HAL Id: hal-01235050

https://hal-centralesupelec.archives-ouvertes.fr/hal-01235050

Submitted on 27 Nov 2015

HAL is a multi-disciplinary open access archive for the deposit and dissemination of scientific research documents, whether they are published or not. The documents may come from teaching and research institutions in France or abroad, or from public or private research centers.
L'archive ouverte pluridisciplinaire HAL, est destinée au dépôt et à la diffusion de documents scientifiques de niveau recherche, publiés ou non, émanant des établissements d'enseignement et de recherche français ou étrangers, des laboratoires publics ou privés. 


\title{
Multirate technique for explicit Discontinuous Galerkin computations of time domain Maxwell equations on complex geometries
}

\author{
A. Kameni ${ }^{1}$, B. Seny ${ }^{2}$, L. Pichon ${ }^{1}$ \\ ${ }^{1}$ Group of Electrical Engineering - Paris, UMR 8507 CNRS, CentraleSupelec, Université Paris Sud, Université Pierre et Marie Curie, \\ Plateau du Moulon, 91192 Gif-Sur-Yvette Cedex, France \\ 2 Institute of Mechanics, Materials and Civil Engineering, Université Catholique de Louvain-la-Neuve, \\ 4-6 avenue Georges Lemaître B-1348, Louvain-la-Neuve, Belgique
}

\begin{abstract}
This paper presents a multirate technique to improve and optimize the time step of a second order 2 -stages Explicit Runge-Kutta scheme (ERK). In this technique, the mesh elements are stored in different groups according to their stable time steps. These groups are sorted into two classes. The bulk groups where the 2-stages ERK method is applied once or repeatedly. The buffer groups that used to accommodate transition between two bulks groups. This technique is proposed for accelerating explicit discontinuous Galerkin computations of time domain Maxwell equations. An application example on human skull is proposed to show the efficiency of this technique to simulate wave propagation on complex geometries.
\end{abstract}

Index Terms-Discontinuous Galerkin method, Explicit Runge-Kutta (ERK) schemes, Maxwell equations, Multirate technique

\section{INTRODUCTION}

$\mathbf{E}$ XPLICIT methods such as the Runge-Kutta schemes (ERK) are commonly used to perform electromagnetic modeling of complex systems. The maximum allowable time step to ensure stability and convergence depends on the CFL (Courant-Friedrichs-Lewy) condition. The time step must be proportional to the element size and inversely proportional to the maximum eigenvalue of the Jacobian of the system. Unstructured meshes are often required to capture a wide spectrum of physical scales. Local mesh refinements may be responsible for significant gaps between the smallest an the average element sizes. In such cases, the efficiency of traditional single rate explicit time stepping methods may be drastically low with respect to the problem size.

The discontinuous Galerkin methods are well suited to be used in combination with a local time stepping strategy to reduce the expensive computations by adapting the time step under local stability conditions [1] [2]. The multirate methods are a subset of the local time-stepping schemes family. They allow the use of different time steps that are integer ratios of each other to solve a discrete system [3]. This technique has been used with a multi-step Adams-Bashforth scheme to improve Discontinuous Galerkin computations of Maxwell equations when the mesh is sorted in two groups [4]. The first is constituted by fine elements where Adams-Bashforth scheme is applied repeatly and the second by coarse elements where Adams-Bashforth scheme is applied once. The development and implementation of ERK multirate schemes have been recently proposed to accelerate geophysical flow computations when the mesh is

Manuscript submitted July 6, 2015. Corresponding author: Abelin Kameni (email: abelin.kameni@geeps.centralesupelec.fr) sorted in few groups [5]. An extension of these strategies to the parallel framework hase also been proposed in [6].

In this paper a multirate technique based on a second order accurate 2-stages ERK method (Heun's method) is presented and applied on Maxwell equations. This approach consists in gathering the mesh elements in different groups that satisfy the CFL condition for a certain range of time steps. These groups are sorted into two classes. The bulk groups where a $2-$ stages ERK method is applied once or repeatedly. The buffer groups which are used to accommodate the transition between two bulk groups and where a 2-stages ERK scheme is adapted to coincide with the ERK schemes used in the bulk groups. Using of these buffer groups allow to increase reduction of computational cost. The efficiency of this approach is shown through computations of an electric field on a human skull.

\section{MAXWELl EQUATIONS AND DiscReTE SYSTEM}

Let $E, H$ and $J$ respectively, be the electric field and the magnetic field and the current density. They satisfy the Maxwell equations given by (1):

$$
\left\{\begin{array}{l}
\epsilon \partial_{t} E-\nabla \times H=-J \\
\mu \partial_{t} H+\nabla \times E=0
\end{array}\right.
$$

where $\epsilon$ and $\mu$ are respectively the permittivity and the permeability of the medium. In a conductive medium, $J=\sigma E$, with $\sigma$ the conductivity.

The Discontinuous Galerkin methods are introduced for solving the conservative form of partial differential equations. This method consists in discretizing the variational formulation of 
(2) on each mesh element $T$ of the domain $\Omega=\cup T$.

$$
\left\{\begin{array}{l}
\int_{T} \epsilon \partial_{t} E \phi-\int_{T} H \times \nabla \phi-\int_{\partial T}(n \times H) \phi=-\int_{T} \sigma E \phi \\
\int_{T} \mu \partial_{t} H \psi+\int_{T} E \times \nabla \psi+\int_{\partial T}(n \times E) \psi=0
\end{array}\right.
$$

where $\phi$ and $\psi$ are test functions.

In each $T$, a Finite Element Method is applied and the mapping technique is used to facilitate the use of high order mesh elements. The interfaces terms are replaced by numerical flux expressions as in a Finite Volume Method. Different formulations of the flux expressions exist [7]. These following expressions (3) resulting in different numerical schemes are implemented. For $\alpha=0$, centered fluxes are obtained and numerical schemes are dispersive. For $\alpha=1$, upwind fluxes are obtained and numerical schemes are dissipative.

$$
\left\{\begin{array}{l}
(n \times H)^{n u m}=n \times \frac{\left\{\sqrt{\frac{\mu}{\epsilon}} H\right\}}{\left\{\sqrt{\frac{\mu}{\epsilon}}\right\}}-\alpha\left(n \times \frac{(n \times[E])}{\left\{\sqrt{\frac{\mu}{\epsilon}}\right\}}\right) \\
(n \times E)^{n u m}=n \times \frac{\left\{\sqrt{\frac{\epsilon}{\mu}} E\right\}}{\left\{\sqrt{\frac{\epsilon}{\mu}}\right\}}+\alpha\left(n \times \frac{(n \times[H])}{\left\{\sqrt{\frac{\epsilon}{\mu}}\right\}}\right)
\end{array}\right.
$$

where $[u]=\frac{u^{+}-u^{-}}{2}$ and $\{u\}=\frac{u^{+}+u^{-}}{2}$, with $u=(E, H)$. The subscript "-" denotes the values for fields in the current element, while " + " is for the adjacent element.

The spatial discretization of Maxwell's equations leads to an ordinary differential equation on each mesh element $T$ that can be presented as a Cauchy problem (4) given by:

$$
\left\{\begin{array}{r}
\frac{d u_{i}}{d t}=f\left(u_{i}(t), t\right) \\
u_{i}(0)=u_{i}^{0}
\end{array}\right.
$$

The explicit Runge-Kutta methods are set to integrate the solution in time.

\section{MultiRATE STRATEGY}

\section{A. Multirate time integration}

In this paper the multirate time integration is based on the Heun's method which is a second order accurate 2-stages ERK method, that we note RK22, whose Butcher tableau is given in TABLE I(Left). It allows to compute the next step solution $u^{n+1}$ from the previous one $u^{n}$ :

$$
\begin{aligned}
(s 1): u^{(1)} & =u^{n} \text { and } K^{1}=f\left(u^{(1)}, t^{n}\right) \\
(s 2): u^{(2)} & =u^{n}+\Delta t K^{1} \text { and } K^{2}=f\left(u^{(2)}, t^{n}+\Delta t\right) \\
u^{n+1} & =u^{n}+\frac{1}{2} \Delta t\left(K^{1}+K^{2}\right)
\end{aligned}
$$

The aim of the multirate time integration is to compute the discrete solution in groups whose stable time steps are different. Let's consider a mesh example in Fig.1(Left). It has two bulk groups of stable time steps $\Delta t_{m}$ and $2 \Delta t_{m}$ respectively noted $R$ and $P$. With a traditional single rate method, the time step $\Delta t_{m}$ is used for all mesh elements. Our goal is to run the elements of group $R$ with time step $\Delta t_{m}$ and those of $P$ with
$2 \Delta t_{m}$ to reduce the computational costs. The idea is to apply once the RK22 on $P$, and twice successively on $R$.
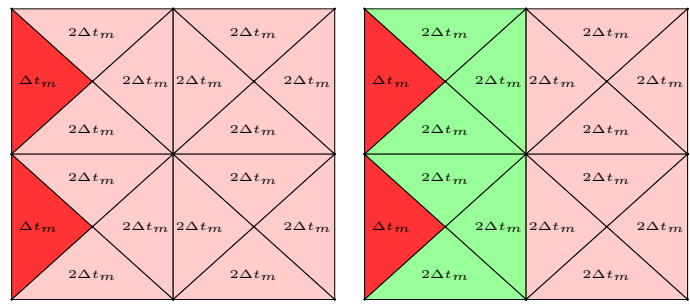

Fig. 1. Example group partitionning for multirate: (Left) Initial bulk groups $P$ with time step $2 \Delta t_{m}$ and $R$ with time step $\Delta t_{m}$, (Right) a buffer group $G$ with time step $2 \Delta t_{m}$ is inserted between the two bulk groups.

The use of RK22 in group $R$ with time step $\Delta t_{m}$ has an influence on the integration scheme in group $P$. This influence is limited at the two connected neighboring elements because the base method has two stages. If RK22 with $2 \Delta t_{m}$ is used on these elements, conservation of the fluxes at interfaces is not satisfied. That is why a buffer region is introduced between groups of different stable time step as shown in Fig.1 (Right) where a buffer group noted $G$ is inserted between the bulk group $P$ and $R$.

Let's denote $u_{0}$ the solution on an element of $R, u_{1}$ the solution on an element of $G$ neighbor of $R, u_{2}$ the solution in an element of $G$ neighbor of $P, u_{3}$ the solution in an element of $P$.

Apply RK22 with time step $\triangle t_{m}$ leads to $u_{0}^{n+\frac{1}{2}}$ :

$$
\begin{aligned}
(s 1): u_{0}^{(1)} & =u_{0}^{n} ; K_{0}^{1}=f\left(u_{0}^{(1)}, t^{n}\right) \\
(s 2): u_{0}^{(2)} & =u_{0}^{n}+\frac{1}{2} \Delta t K_{0}^{1} ; K_{0}^{2}=f\left(u_{0}^{(2)}, t^{n}+\frac{1}{2} \Delta t\right) \\
u_{0}^{n+\frac{1}{2}} & =u_{0}^{n}+\frac{1}{4} \Delta t\left(K_{0}^{1}+K_{0}^{2}\right)
\end{aligned}
$$

Apply again RK22 allows to compute $u_{0}^{n+1}$ :

$$
\begin{aligned}
(s 3): u_{0}^{(3)} & =u_{0}^{n+\frac{1}{2}} ; K_{0}^{3}=f\left(u_{0}^{(3)}, t^{n+\frac{1}{2}}\right) \\
(s 4): u_{0}^{(4)} & =u_{0}^{n+\frac{1}{2}}+\frac{1}{2} \Delta t K_{0}^{3} ; K_{0}^{4}=f\left(u_{0}^{(4)}, t^{n+\frac{1}{2}}+\frac{1}{2} \Delta t\right) \\
u_{0}^{n+1} & =u_{0}^{n}+\Delta t\left(\frac{1}{4} K_{0}^{1}+\frac{1}{4} K_{0}^{2}+\frac{1}{4} K_{0}^{3}+\frac{1}{4} K_{0}^{4}\right)
\end{aligned}
$$

These four steps constitute a 4-stages ERK method noted RK22 ${ }^{4 a}$ stable for the time step $\Delta t$ whose Butcher tableau is given by TABLE I(Center).

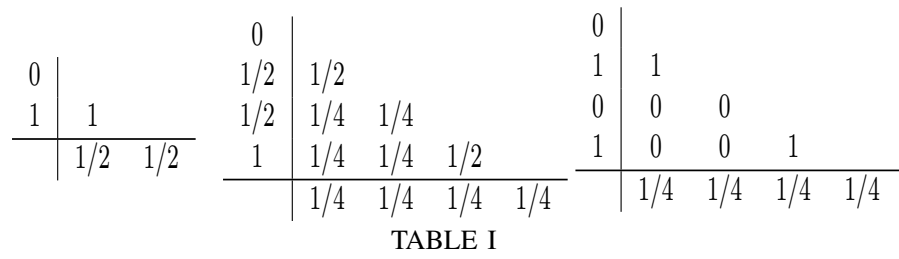

(LEFT) BUTCHER TABLEAU OF RK22 (CENTER) BUTCHER TABLEAU OF RK22 ${ }^{4 a}$ ISSUED FROM APPLYING TWICE RK22, (RIGHT) BUTCHER TABLEAU OF THE EXTENDED METHOD RK $22^{4 b}$.

Since RK22 ${ }^{4 a}$ has four stages, the base method RK22 is extended to a four stages method noted RK22 ${ }^{4 b}$, stable for the time step $\Delta t$, whose Butcher tableau is given by TABLE 
I(Right). For elements of $P$ whose integration schemes are not influenced by the use of $\operatorname{RK} 22^{4 a}$, the solution $u_{3}^{n+1}$ is computed by RK22 ${ }^{4 b}$ in four stages:

$$
\begin{aligned}
(s 1): u_{3}^{(1)} & =u_{3}^{n} ; K_{3}^{1}=f\left(u_{3}^{(1)}, t^{n}\right) \\
(s 2): u_{3}^{(2)} & =u_{3}^{n}+\Delta t K_{3}^{1} ; K_{3}^{2}=f\left(u_{3}^{(2)}, t^{n}+\Delta t\right) \\
(s 3): u_{3}^{(3)} & =u_{3}^{(1)} ; K_{3}^{3}=K_{3}^{1} \\
(s 4): u_{3}^{(4)} & =u_{3}^{(2)} ; K_{3}^{4}=K_{3}^{2} \\
u_{3}^{n+1} & =u_{3}^{n}+\Delta t\left(\frac{1}{4} K_{3}^{1}+\frac{1}{4} K_{3}^{2}+\frac{1}{4} K_{3}^{3}+\frac{1}{4} K_{3}^{4}\right)
\end{aligned}
$$

Solutions in the buffer group $G$ are obtained by using coefficients of the Butcher tableau TABLE I(Right).

The solution $u_{1}^{n+1}$ is obtained with:

$$
\begin{aligned}
(s 1): u_{1}^{(1)} & =u_{1}^{n} ; K_{1}^{1}=f\left(u_{1}^{(1)}, t^{n}\right) \\
(s 2): u_{1}^{(2)} & =u_{1}^{n}+\Delta t K_{1}^{1} ; K_{1}^{2}=f\left(u_{1}^{(2)}, t^{n}+\Delta t\right) \\
(s 3): u_{1}^{(3)} & =u_{1}^{(1)} ; K_{1}^{3} \neq K_{1}^{1} \\
(s 4): u_{1}^{(4)} & =u_{1}^{(3)}+\Delta t K_{1}^{3} ; K_{1}^{4}=f\left(u_{1}^{(4)}, t^{n}+\Delta t\right) \\
u_{1}^{n+1} & =u_{1}^{n}+\Delta t\left(\frac{1}{4} K_{1}^{1}+\frac{1}{4} K_{1}^{2}+\frac{1}{4} K_{1}^{3}+\frac{1}{4} K_{1}^{4}\right)
\end{aligned}
$$

Note that $K_{1}^{3} \neq K_{1}^{1}$ because it depends on interface term with an element of group $R$ whose stage (s3) in (7) is computed at $t^{n+\frac{1}{2}}$.

The solution $u_{2}^{n+1}$ is obtained with:

$$
\begin{aligned}
(s 1): u_{2}^{(1)} & =u_{2}^{n} ; K_{2}^{1}=f\left(u_{2}^{(1)}, t^{n}\right) \\
(s 2): u_{2}^{(2)} & =u_{2}^{n}+\Delta t K_{2}^{1} ; K_{2}^{2}=f\left(u_{2}^{(2)}, t^{n}+\Delta t\right) \\
(s 3): u_{2}^{(3)} & =u_{2}^{(1)} ; K_{2}^{3}=K_{2}^{1} \\
(s 4): u_{2}^{(4)} & =u_{2}^{(2)} ; K_{2}^{4} \neq K_{2}^{2} \\
u_{2}^{n+1} & =u_{2}^{n}+\Delta t\left(\frac{1}{4} K_{2}^{1}+\frac{1}{4} K_{2}^{2}+\frac{1}{4} K_{2}^{3}+\frac{1}{4} K_{2}^{4}\right)
\end{aligned}
$$

In this case $K_{2}^{4} \neq K_{2}^{2}$ because it depends on interface term with an element of group $G$ whose stage (s4) in (9) has been influenced by stage $(s 3)$ in (7) computed at $t^{n+\frac{1}{2}}$.

The RK22 ${ }^{4 a}$ and RK22 ${ }^{4 b}$ have the same number of stages and the same weighting coefficients as shown in TABLE I. This allows to preserve fluxes conservation at interfaces between theses two groups. The resulting multirate scheme is a second order accurate method stable for the time step $\Delta t=2 \Delta t_{m}$.

\section{B. Construction of the multirate groups}

Let's consider a mesh of the domain defined by the tessellation $\Omega=\cup T$. In this paper, the multirate groups are built for having their stable time steps of ratio $\kappa=2$. Let's note $\delta t_{m}$ and $\delta t_{M}$ respectively the time steps according to CFL of the smallest and the biggest elements. The mesh elements are gathered in different groups that satisfy the CFL condition for a certain range of time steps.

If the time step of the multirate strategy is $\Delta t \in\left[\Delta t_{m}, \Delta t_{M}\right]$, the number of multirate groups $N_{g}=z_{g}+1$ where $z_{g}$ is an integer defined by $z_{g}=\log _{2}\left(\frac{\Delta t}{\Delta t_{m}}\right)$. The multirate group number 0 is constituted by elements whose time steps are in $\left[\Delta t, \Delta t_{M}\right]$. The multirate group number $z$ is constituted by elements whose time steps are in $\left[\frac{\Delta t}{2^{z+1}}, \frac{\Delta t}{2^{z}}\right]$, with $z \leq z_{g}$. The time step of the multirate strategy $\Delta t$ that guarantee stability of the method has to be chosen such that $\Delta t \in\left[\Delta t_{m}, 2^{z} \Delta t_{m}\right]$ where $2^{z} \Delta t_{m} \leq \Delta t_{M}$.

For construction of multirate groups we choose in a first
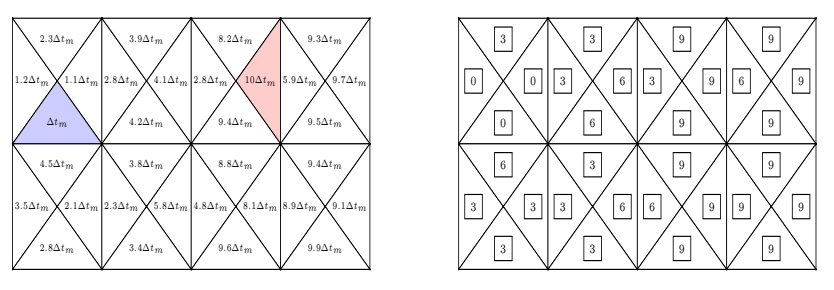

Fig. 2. Example of sorting of mesh elements into different groups: (Left) The time steps of each elements according to their CFL condition, (Right) Sorting of elements in 4 groups of tags $0,3,6$ and 9 .

step to assign a $\operatorname{tag} \theta=3\left(z_{g}-z\right)$ at each group. The example mesh presented in Fig.2 shows the organization of the elements in 4 multirate groups. The tag 9 is assigned to group number 0 whose elements have stable time steps in the interval $\left[8 \Delta t_{m}, 10 \Delta t_{m}\right]$. The tag 6 is assigned to group number 1 whose elements have stable time steps in the interval $\left[4 \Delta t_{m}, 8 \Delta t_{m}[\right.$. The tag 3 is assigned to group number 2 whose elements have stable time steps in the interval $\left[2 \Delta t_{m}, 4 \Delta t_{m}[\right.$. The tag 0 is assigned to group number 3 whose elements have stable time steps in the interval $\left[\Delta t_{m}, 2 \Delta t_{m}[\right.$.

The next step is to introduce the buffer groups that serve to accommodate transition between bulk groups. These buffers have a size of two connected elements because the base method ERK22 has two stages. To distinguish groups, the $\operatorname{tag} \theta=3\left(z_{g}-z\right)+2$ is attributed to buffer groups and the $\operatorname{tag} \theta=3\left(z_{g}-z\right)$ is conserved for bulk groups. The group of tag 0 remains the same, and the buffer elements are successively introduced. During this process, it is ensured that two neighboring elements have neighboring tags.
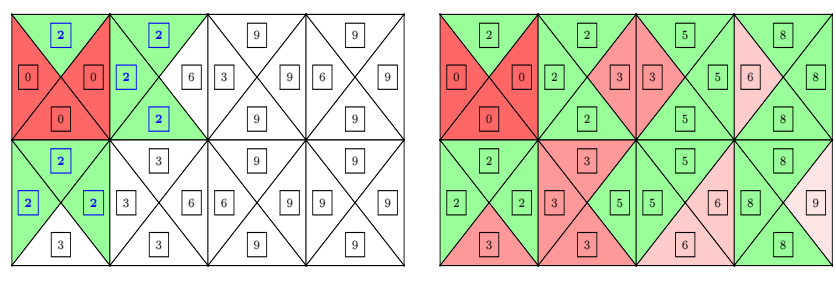

Fig. 3. Introduction of buffer groups : (Left) The first buffer elements are introduced around the group of tag 0, (Right) Four bulk groups of tag 0, 3, 6,9 and three buffer groups of tag $2,5,8$ are obtained.

The illustration presented in Fig. 3 shows the introduction of buffer elements. The first buffer elements are introduced around the bulk group of tag 0 . Four bulk groups of tag 0,3 , 6,9 and three buffer groups of tag $2,5,8$ are constituted. 

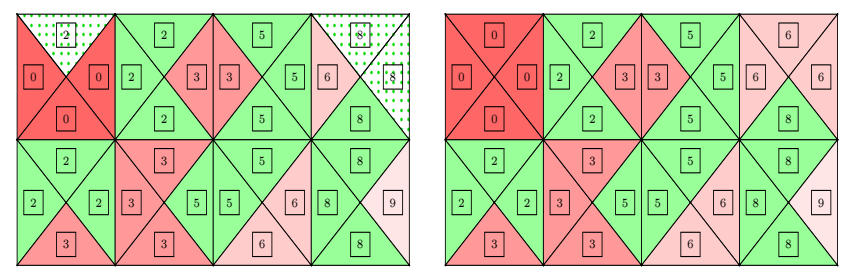

Fig. 4. (Left) Location of the unnecessary buffer elements; (Right) The final multirate partitionning.

The last step consists in transferring the unnecessary buffer elements to the bulk group with inferior tag. These elements which appear at the boundary and are not located between two bulk groups. Fig.4 shows the unnecessary buffers elements corresponding to groups of tag 2 and 8 (dotted elements) which are transferred to groups 0 and 6 .

\section{NUMERICAL EXAMPLE}

The simulation domain is a box $B\left(\epsilon_{B}=\epsilon_{0}, \sigma_{B}=0 S / m\right)$ containing a skull $S_{K}\left(\epsilon_{S_{K}}=32 \epsilon_{0}, \sigma_{S_{K}}=0.8 S / \mathrm{m}\right)$ which is inside a sphere $S_{1}\left(\epsilon_{S_{1}}=14 \epsilon_{0}, \sigma_{S_{1}}=0.1 \mathrm{~S} / \mathrm{m}\right)$ as shown in Fig.5. Inside the skull there is another sphere $S_{2}\left(\epsilon_{S_{2}}=49 \epsilon_{0}\right.$, $\sigma_{S_{2}}=1.15 \mathrm{~S} / \mathrm{m}$ ). The permeability of vacuum $\mu_{0}$ is assumed in all the domain.

The faces of the box oriented along the $y$-axis are magnetic walls and those oriented along the $z$-axis are electric walls. On the left side, an absorbing Silver-muller boundary condition is set. An incident field $\vec{E}_{\text {inc }}=\left(0,0, E_{z}^{\text {inc }}\right)$ propagates from the right side. It is a Gaussian modulated pulse of center frequency $f_{0}=1.2 G h z$ such that: $E_{z}^{i n c}(t)=\sin \left(2 \pi f_{0} t\right) e^{-a\left(t-t_{0}\right)^{2}}$, $t_{0}=2.5 \mathrm{~ns}, a=15.10^{8}$. The simulations are carried out for a duration $T_{f}=25 \mathrm{~ns}$. They are performed on 8 cores $-15 G o /$ Ram $-2.3 G H z$ computer.

A tetrahedral mesh of 279802 third order spatial elements is used. The minimum and maximum element-wise stable time steps according to the CFL condition are respectively $\Delta t_{m}=24 \cdot 10^{-14} \mathrm{~s}$ and $\Delta t_{M}=17 \cdot 10^{-12} \mathrm{~s}$. The single rate RK22 and the LeapFrog scheme are firstly used with $\Delta t_{m}$. Their CPU times are $\mathrm{CPU}_{R K 22}=254100 \mathrm{~s}$, $\mathrm{CPU}_{L F}=140100 s$. They are compared to that obtained with the multirate strategy in TABLE II to show the computational gain. The set up of the groups takes few seconds which are neglected in the CPU times. When $N_{g}$ increases, the percentage of buffer elements also increases and the gain is improved. The higher stable time step is $2^{5} \Delta t_{m}$ because $2^{6} \Delta t_{m}>\Delta t_{M}$. The electric field recorded at the center of the sphere $S_{2}$ is plotted in Fig.6 to show that results of the three methods are very close.

\section{CONClusion}

In this paper a multirate technique to reduce the computational cost of explicit computations of time domain Maxwell

\begin{tabular}{|c|c|c|c|c|c|}
\hline$N_{g}$ & $\Delta t(s)$ & buffer elts & $\mathrm{CPU}_{t o t}(s)$ & Gain $_{L F}$ & Gain $_{R K 22}$ \\
\hline 2 & $2 \Delta t_{m}$ & $1 \%$ & 137100 & 1.02 & 1.85 \\
\hline 3 & $2^{2} \Delta t_{m}$ & $14 \%$ & 71100 & 1.97 & 3.57 \\
\hline 4 & $2^{3} \Delta t_{m}$ & $34 \%$ & 49200 & 2.84 & 5.16 \\
\hline 5 & $2^{4} \Delta t_{m}$ & $38 \%$ & 45200 & 3.09 & 5.62 \\
\hline 6 & $2^{5} \Delta t_{m}$ & $39 \%$ & 42040 & 3.33 & 6.04 \\
\hline
\end{tabular}

TABLE II

CPU TIME OF MULTIRATE SIMULATIONS AND THE GAINS VERSUS LEAPFROG SCHEME $\left(\right.$ GAIN $\left._{L F}\right)$ AND THE SINGLE RATE SCHEME $\left(\right.$ GAIN $\left._{R K 22}\right)$.
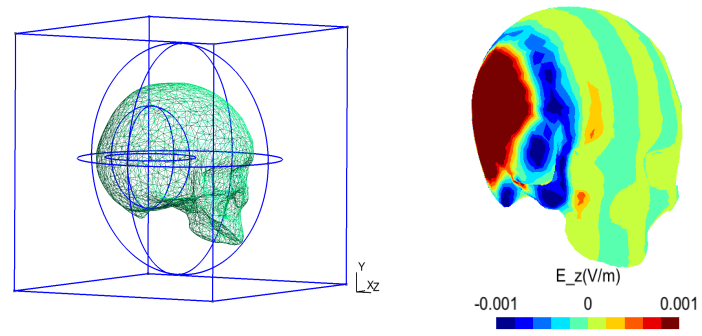

Fig. 5. (left) Simulation domain (right) Illustration of the electric field that propagates.

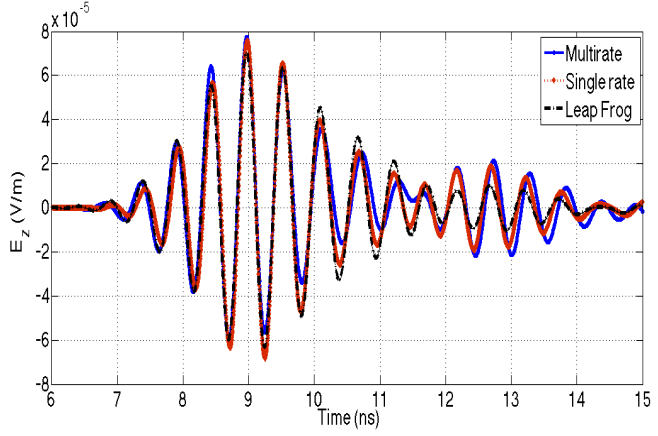

Fig. 6. Comparison of $E_{z}$ recorded at the center of the sphere $S_{2}$.

equations on complex geometries is presented. A numerical example shows the gain obtained compared to simulations performed with the classical LeapFrog and Heun methods.

\section{REFERENCES}

[1] Schomann, S.; Warburton, T.; Clemens, M.; Local Timestepping Techniques Using Taylor Expansion for Modeling Electromagnetic Wave Propagation With Discontinuous Galerkin-FEM, IEEE Trans. Magn, vol. 46, num. 8, pp.3504-3507, 2010.

[2] S. Descombes, S.; Lanteri, S.; Moya, L.; Locally Implicit Time Integration Strategies in a Discontinuous Galerkin Method for Maxwell's Equations, J. Sc. Com., vol 56, num. 1, pp.190-218, 2013

[3] Constantinescu, E.M.; Sandu, A.; Multirate time stepping methods for hyperbolic conservation laws, Journal of Scientific Computing, Vol.33, pp. 239-278, 2007.

[4] Godel, N.; Schomann, S.; Warburton, T.; Clemens, M.; GPU Accelerated Adams-Bashforth Multirate Discontinuous Galerkin FEM Simulation of High-Frequency Electromagnetic Fields, IEEE Trans. Magn, vol. 46, num. 8, pp.2735-2738, 2010.

[5] Seny, B. and al., Multirate time stepping for accelerating explicit discontinuous Galerkin computations with application to geophysical flows, Inter. J. Num. Meth in fluids, vol. 71, pp.41-64, 2013.

[6] B. Seny and al., An efficient parallel implementation of explicit multirate Runge-Kutta schemes for discontinuous Galerkin computations, Journal of Computational Physics Vol. 256, pp.135-160, 2014.

[7] L. Fezoui, S. Lanteri, S. Lohrengel, S. Piperno Convergence and Stability of a Discontinuous Galerkin Time-Domain Method for the 3D Heterogeneous Maxwell Equations on Unstructured Meshes, ESAIM-M2AN, Vol 39, number 6, pp. 1149-1176, 2005. 